Tôhoku Math. Journ.

25 (1973), 53-60.

\title{
TOPOLOGIES ON GROUPS AND A CERTAIN $L$-IDEAL OF MEASURE ALGEBRAS
}

\author{
KeIJI IzUChI AND Tetsuhiro ShIMIzU
}

(Received July 19, 1972)

1. Introduction. Let $G_{\tau_{0}}$ be a non-discrete locally compact abelian group with a topology $\tau_{0}$. Let $M\left(G_{\tau_{0}}\right)$ be the commutative semisimple Banach algebra consists of bounded regular Borel measures on $G_{\tau_{0}}$. We write $\mathfrak{M}$ the maximal ideal space of $M\left(G_{\tau_{0}}\right)$. For $\mu \in M\left(G_{\tau_{0}}\right)$, we put $\mu^{*}(E)=$ $\overline{\mu(-E)}$ for all Borel subset $E$ of $G_{\tau_{0}}$. Then we have $\mu^{*} \in M\left(G_{\tau_{0}}\right)$ and $M\left(G_{\tau_{0}}\right)$ is considered a Banach *-algebra. Let $\Delta$ be the set of all symmetric multiplicative linear functionals on $M\left(G_{\tau_{0}}\right)$, that is $\Delta=\left\{f \in \mathfrak{M}: f\left(\mu^{*}\right)=\right.$ $\overline{f(\mu)}$ for all $\left.\mu \in M\left(G_{\tau_{0}}\right)\right\}$. A closed subspace (subalgebra, ideal) $N$ of $M\left(G_{\tau_{0}}\right)$ will be called an $L$-subspace ( $L$-subalgebra, $L$-ideal) if $N$ satisfies the condition; $\mu \in M\left(G_{\tau_{0}}\right), \nu \in N$ and $\mu$ is absolutely continuous with respect to $\nu$, then $\mu \in N$. For a subspace $N$ of $M\left(G_{\tau_{0}}\right)$, we put $N^{\perp}=\left\{\mu \in M\left(G_{\tau_{0}}\right): \mu\right.$ is mutually singular with $\nu \in N\}$.

In this note, we consider the following subspace of $M\left(G_{\tau_{0}}\right) ; M(\Delta)=$ $\left\{\mu \in M\left(G_{\tau_{0}}\right): \hat{\mu}(f)=0\right.$ for all $\left.f \notin \Delta\right\}$. J. H. Williamson ([9]) showed that for every $\mu \in M(\Delta),\left|\hat{\mu}_{d}(f)\right|<\left|\hat{\mu}_{c}(f)\right|$ for all $f \in \mathfrak{M}$, where $\mu_{d}$ and $\mu_{c}$ are the discrete part and the continuous part of $\mu$, respectively. And he conjectured that $\mu_{d}=0$ for every $\mu \in M(\Delta)$ ([9]). Using the results of J. L. Taylor ([7]), T. Shimizu ([6]) showed that $M(\Delta)$ is a proper $L$-ideal of $M\left(G_{\tau_{0}}\right)$ and Williamson's conjecture is true. For a locally compact group topology $\tau$ on $G$ which is strictly stronger than $\tau_{0}$, we may consider $M\left(G_{\tau}\right)$ a prime $L$-subalgebra of $M\left(G_{\tau_{0}}\right)$ with natural injection ([3]). It is clear that $M_{c}\left(G_{\tau_{0}}\right)^{\perp}=M\left(G_{\tau_{d}}\right)$, where $\tau_{d}$ is the discrete topology on $G$ and $M_{c}\left(G_{\tau_{0}}\right)=\left\{\mu \in M\left(G_{\tau_{0}}\right): \mu\right.$ is continuous $\}$. From the above fact, we have the following conjecture:

Conjecture I. $M(\Delta)$ is contained in $M\left(G_{\tau}\right)^{\perp}$.

For $M\left(G_{\tau}\right)$, there is a Raikov system $\mathfrak{F}$ such that $M\left(G_{\tau}\right)=M(\mathfrak{F})$, where $M(\mathfrak{F})=\left\{\mu \in M\left(G_{\tau_{0}}\right)\right.$ : there is $A \in \mathfrak{F}$ such that $\mu$ is concentrated on $\left.A\right\}$. Thus we have a more generally conjecture as follows:

Conjecture II. For a proper Raikov system $\mathfrak{F}$, we have $M(\Delta) \subset M(\mathfrak{F})^{\perp}$. In $\S 1$, we show that our conjecture II is true, if $G_{\tau_{0}}$ is metrizable. In $\S 2$, we show that our conjecture $I$ is true. In $\S 3$, we show a property of the 
Gelfand transforms of $M(\Delta)$, using Taylor's structure semigroup of $M\left(G_{\tau_{0}}\right)$.

2. Metrizable group. Throughout this section, let $G$ be a non-discrete locally compact abelian group. A subset of $G$ is called type $F_{\sigma}$ if it is a countable union of compact subsets of $G$. A collection of subsets of $G$ of type $F_{\sigma}$ is called a Raikov system if the following properties hold:

(1) If $A_{1} \in \mathfrak{F}$ and $A_{2}$ is a subset of $A_{1}$ of type $F_{\sigma}$, then $A_{2} \in \mathfrak{F}$.

(2) The union of a countable collection of sets in $\mathfrak{F}$ also in $\mathfrak{F}$.

(3) If $A \in \mathfrak{F}$ and $t \in G$, then $A-t \in \mathfrak{F}$.

(4) If $A \in \mathfrak{F}$, then $A+A \in \mathfrak{F}$.

Let $m$ be a Haar measure on $G$. A Raikov system $\mathfrak{F}$ such that $m(A)=0$ for every $A \in \mathfrak{F}$, will be called proper. For a $\sigma$-compact subset $A$, there exists a minimal Raikov system containing $A$. Such a Raikov system will be called a single generated Raikov system. For a Raikov system $\mathfrak{F}$, we put $M(\mathfrak{F})=\{\mu \in M(G)$ : there exists $A \in \mathfrak{F}$ such that $\mu$ is concentrated on $A\}$. For a Raikov system $\mathfrak{F}$, if $A \in \mathfrak{F}$ implies $-A \in \mathfrak{F}$, then $\mathfrak{F}$ is a symmetric Raikov system. For a single generated symmetric Raikov $\mathfrak{F}$, there is a group which generates $\mathfrak{F}$.

J. L. Taylor ([7]) showed that there exists a compact topological semigroup $S$ and an isometric isomorphism $\theta$ from $M(G)$ into $M(S)$ such that the image of $\theta$ is weak* dense in $M(S)$ and the maximal ideal space of $M(G)$ is identified with the set $\hat{S}$ of all continuous semicharacters on $S$. For $\mu \in M(G)$, the Gelfand transform $\hat{\mu}$ of $\mu$ is given by $\hat{\mu}(f)=\int_{S} f d \theta \mu$ for every $f \in \hat{S}$.

For a given subset $E$ of $G$ which contains 0 , we shall say a subset $F$ of $G$ is $(E, 1)$-independent if the following relation holds:

$\sum_{r=1}^{N} n_{r} x_{r} \in E$ if and only if $n_{r}=0$ for $1 \leqq r \leqq N$, where $x_{1}, \cdots, x_{N}$ are distinct elements of $F$ and $n_{1}, \cdots, n_{N}$, are integers with $\left|n_{r}\right| \leqq 1$.

THEOREM 1. Let $\mathfrak{F}$ be a proper symmetric Raikov system with a single generator. Let $H$ be a group which generates $\mathfrak{F}$. If there exists a perfect compact $(H, 1)$-independent set $P$, then we have $M(\Delta) \subset M(\mathfrak{F})^{\perp}$.

Proof. Let $\mu_{0}$ be a positive continuous measure concentrated on $P$, with $\left\|\mu_{0}\right\|=1$. We put $\mu=(1 / 2)\left(\mu_{0}+\mu_{0}^{*}\right)$, then $\mu=\mu^{*}$ and $\mu$ is concentrated on $Q=P \cup(-P)$. For a non-negative measure $\omega_{0} \in M(\mathfrak{\Im})$ with $\left\|\omega_{0}\right\|=1$, we put $\omega=(1 / 2)\left(\omega_{0}+\omega_{0}^{*}\right)$ and $\sigma=\omega^{2}-\mu^{2}$. As the proof of Proposition 2 of [10] we obtain that $\mu^{n_{1}} \omega^{m_{1}} \perp \mu^{n_{2}} \omega^{m_{2}}$ for $\left(n_{1}, m_{1}\right) \neq\left(n_{2}, m_{2}\right)$ where $n_{i}, m_{i}(i=1,2)$ are positive integers. So we have

$$
\left\|\sigma^{n}\right\|=\left\|\sum_{k=0}^{n}\left(\begin{array}{l}
n \\
k
\end{array}\right)(-1)^{k} \mu^{2 k} \omega^{2(n-k)}\right\|=\sum_{k=0}^{n}\left(\begin{array}{l}
n \\
k
\end{array}\right)\left\|\mu^{2 k} \omega^{2(n-k)}\right\|=\sum_{k=0}^{n}\left(\begin{array}{l}
n \\
k
\end{array}\right)=2,
$$


and the spectral norm of $\sigma$ is 2 . Hence there is a complex homomorphism $h$ of $M(G)$ such that $|h(\sigma)|=2$. Since $\|\mu\|=1$, we have that $\left|h\left(\mu^{2}\right)\right| \leqq 1$, and $\left|h\left(\omega^{2}\right)-h\left(\mu^{2}\right)\right|=|h(\sigma)|=2$ if and only if $h\left(\omega^{2}\right)=-h\left(\mu^{2}\right)$ and $|h(\omega)|=|h(\mu)|=1$. This shows that $h$ is non-symmetric. Let $f$ be a continuous semicharacters on $S$ such that $h(\lambda)=\int_{S} f d \theta \lambda$ for every $\lambda \in M(G)$. Since $\|\omega\|=1,|f| \leqq 1$ and $|h(\omega)|=1$, we obtain $\operatorname{supp} \theta \omega \subset\{x \in S:|f(x)|=1\}$. By Shimizu [6], we have $\omega \in M(\Delta)^{\perp}$. Then $M(\Delta) \subset M(\mathfrak{F})^{\perp}$.

q.e.d.

COROLlaRY 2. If $G$ is metrizable, then we have $M(\Delta) \subset M(\mathfrak{F})^{\perp}$ for $a$ proper Raikov system $\mathfrak{F}$.

Proof. For any $\mu \in M(\mathfrak{F})$, there exists a single generated Raikov system $\mathfrak{F}_{0}$ such that $\mu \in M\left(\mathfrak{F}_{0}\right)$ and $M\left(\mathfrak{F}_{0}\right) \subset M(\mathfrak{F})$. If $\mathfrak{F}_{0}$ is a non-symmetric Raikov system, we can easily see $M(\Delta) \subset M\left(\mathfrak{F}_{0}\right)^{\perp}$. If $\mathfrak{F}_{0}$ is a symmetric Raikov system, there is a group $H$ that generates $\mathfrak{\mho}_{0}$. Then there is a perfect compact $(H, 1)$-independent set $P$ as in the proof of Proposition 1 of [10]. By Theorem 1, we have $M(\Delta) \subset M\left(\mathfrak{\mho}_{0}\right)^{\perp}$. Thus we have $M(\Delta) \subset$ $M(\mathfrak{F})^{\perp} \cdot$

q.e.d.

3. Topologies on groups and $M(\Delta)$. Let $G$ be a non-discrete locally compact abelian group and $\hat{G}$ be the dual group of $G$. Let $H$ be a closed subgroup of $G$ and $\varphi$ the canonical continuous homomorphism from $G$ onto $G / H$.

Proposition 3. We put $\Phi \mu(E)=\mu\left(\Phi^{-1}(E)\right)$ for every Borel set $E$ of $G / H$. Then we have the followings:

(a) $\Phi$ is a norm decreasing positive homomorphism from $M(G)$ onto $M(G / H)$.

(b) For every non-negative measure $\nu \in M(G / H)$, there exists a nonnegative measure $\mu \in M(G)$ such that $\Phi \mu=\nu$.

( c ) $\Phi\left(\mu^{*}\right)=(\Phi \mu)^{*}$ for every $\mu \in M(G)$.

Proof. At first, we shall show (a). For every Borel subset $E$ of $G / H$, we have

$$
\int_{G / H} \chi_{E}(y) d \Phi \mu(y)=\int_{G} \chi_{E}(\varphi(x)) d \mu(x)
$$

for every $\mu \in M(G)$, where $\chi_{E}$ is a characteristic function of $E$. Then for every Borel function $f$ on $G / H$, we have

$$
\int_{G / H} f(y) d \Phi \mu(y)=\int_{G} f(\varphi(x)) d \mu(x)
$$

for every $\mu \in M(G)$. Let $\Lambda$ be the annihilator of $H$, then we may consider $\Lambda$ as the dual group of $G / H$. By (5), we have $(\Phi \mu)(\gamma)=\hat{\mu}(\gamma)$ for every 
$\gamma \in \Lambda$. Then we get $(\Phi(\mu * \nu) \hat{)}(\gamma)=(\Phi \mu * \Phi \nu)(\gamma)$ for every $\gamma \in \Lambda$. From the uniqueness theorem, we obtain $\Phi(\mu * \nu)=\Phi \mu * \Phi \nu$. By the definition of $\Phi, \Phi$ is a positive norm decreasing linear mapping. Moreover, $\Phi$ is an onto mapping by ([5]; p. 54). Thus (a) is proved. (c) is clear by the definition. Finally, we shall show (b). For every non-negative measure $\nu \in M(G / H)$, there exists $\mu_{0} \in M(G)$ such that $\Phi \mu_{0}=\nu$. Let $\mu_{0}=\mu_{1}-\mu_{2}+$ $i\left(\mu_{3}-\mu_{4}\right)$ be the Jordan decomposition of $\mu_{0}$, where $\mu_{i} \geqq 0(n=1,2,3,4)$. Since $\Phi \mu_{0} \geqq 0$, we have $\Phi \mu_{0}=\Phi \mu_{1}-\Phi \mu_{2}$. Since $\Phi \mu_{2} \geqq 0$, we get $\Phi \mu_{1} \geqq$ $\Phi \mu_{0} \geqq 0$. Then from Radon-Nikodym's theorem, there exists a non-negative Borel measurable function $f \in L^{1}\left(\Phi \mu_{1}\right)$ such that $\|f\|_{\infty} \leqq 1$ and $\Phi \mu_{0}=f \Phi \mu_{1}$. We put

$$
\mu(E)=\int_{E} f(\varphi(x)) d \mu_{1}(x)
$$

for every Borel subset $E$ of $G$. Then $\mu$ is a non-negative measure on $G$. By (5) and (6), we get

$$
\begin{aligned}
\Phi \mu(A) & =\int_{G} \chi_{A}(\varphi(x)) f(\varphi(x)) d \mu_{1}(x)=\int_{G / H} \chi_{A} \cdot f d \Phi \mu_{1} \\
& =\int_{G / H} \chi_{A} d \Phi \mu_{0}=\nu(A)
\end{aligned}
$$

for every Borel subset $A$ of $G / H$. Thus we have $\Phi \mu=\nu$.

q.e.d.

Proposition 4. Let $H$ be a $\sigma$-compact closed subgroup of $G$. If $E$ is a $\sigma$-compact subset of $G / H$, then $\varphi^{-1}(E)$ is a $\sigma$-compact subset of $G$.

Proof. Without loss of generality, we may assume that $E$ is compact. Let $H$ be a $\sigma$-compact open subgroup of $G$. Since $\varphi\left(H_{0}\right)$ is open, there exists a finite set $\left\{x_{1}, \cdots, x_{n}\right\} \subset G$ such that $E \subset \bigcup_{k=1}^{n}\left(\varphi\left(x_{k}\right)+\varphi\left(H_{0}\right)\right)$. Then we have $\varphi^{-1}(E) \subset \bigcup_{k=1}^{n}\left(x_{k}+H_{0}+H\right)$. Since $H_{0}$ and $H$ are $\sigma$-compact, $H_{0}+H$ is $\sigma$-compact. Then $\bigcup_{k=1}^{n}\left(x_{k}+H_{0}+H\right)$ is $\sigma$-compact. Since $\varphi^{-1}(E)$ is a closed set, $\varphi^{-1}(E)$ is $\sigma$-compact.

q.e.d.

Let $G_{\tau_{0}}$ be an abelian group $G$ with a non-discrete locally compact abelian group topology $\tau_{0}$. Let $\tau$ be a locally compact abelian group topology on $G$ strictly stronger than $\tau_{0}$. Now we consider that $G_{\tau_{0}}$ and $\tau$ are fixed. Let $\eta$ be the continuous identity mapping from $G_{\tau}$ to $G_{\tau_{0}}$. For $\mu \in M\left(G_{\tau}\right)$, we put $\Psi \mu$ the restriction of $\mu$ to the Borel field of $G_{\tau_{0}}$. Then $\Psi$ is an isometric isomorphism from $M\left(G_{\tau}\right)$ into $M\left(G_{\tau_{0}}\right)$ and we may consider that $M\left(G_{\tau}\right)$ is a prime $L$-subalgebra of $M\left(G_{\tau_{0}}\right)$. The following proposition is important for our purpose.

Proposition 5 (J. Inoue [3]). For $\mu \in M\left(G_{\tau_{0}}\right), \mu \in M\left(G_{\tau}\right)$ if and only if there exists a Borel set $C$ of $G_{\tau_{0}}$ such that $\eta^{-1}(C)$ is a $\sigma$-compact subset 
of $G_{\tau}$ and $\mu$ is concentrated on $C$.

CoRollary 6. For $\mu \in M\left(G_{\tau_{0}}\right), \mu \in M\left(G_{\tau}\right)^{\perp}$ if and only if $\mu(C)=0$ for every Borel set $C$ of $G_{\tau_{0}}$ such that $\eta^{-1}(C)$ is $\sigma$-compact.

Let $H$ be a closed subgroup of $G_{\tau_{0}}$, and $\varphi_{1}, \varphi_{2}$ be the canonical homomorphisms from $G_{\tau_{0}}$ onto $G_{\tau_{0}} / H$, from $G_{\tau}$ onto $G_{\tau} / H$, respectively. Let $\psi$ be a continuous identity mapping from $G_{\tau} / H$ to $G_{\tau_{0}} / H$. Then we have the following commutative diagram.

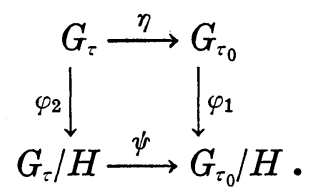

Let $\Phi$ be a canonical homomorphism from $M\left(G_{\tau_{0}}\right)$ onto $M\left(G_{\tau_{0}} / H\right)$ induced by $\varphi_{1}$. The following proposition is followed by Lebesgue's decomposition theorem.

Proposition 7. Let $N$ be an L-subspace of $M\left(G_{\tau_{0}}\right)$, then $N^{\perp}$ is an $L$-subspace and $M\left(G_{\tau_{0}}\right)=N \oplus N^{\perp}$.

Proposition 8. Suppose $H$ is a closed subgroup of $G_{\tau_{0}}$ and a $\sigma$ compact subset of $G_{\tau}$. Then we have

( 7 ) $\Phi\left(M\left(G_{\tau}\right)\right)=M\left(G_{\tau} / H\right)$ and

(8) $\Phi\left(M\left(G_{\tau}\right)^{\perp}\right)=M\left(G_{\tau} / H\right)^{\perp}$.

Proof. At first, we shall show (7). Let $\mu \in M\left(G_{\tau}\right)$, then by Proposition 5 there exists a Borel set $C$ of $G_{\tau_{0}}$ such that $\eta^{-1}(C)$ is $\sigma$-compact and $\mu$ is concentrated on $C$. Then $\Phi \mu$ is concentrated on $\varphi_{1}(C)$. Since $\psi^{-1}\left(\varphi_{1}(C)\right)=$ $\varphi_{2}\left(\eta^{-1}(C)\right)$ and $\eta^{-1}(C)$ is a $\sigma$-compact subset of $G_{\tau}, \psi^{-1}\left(\varphi_{1}(C)\right)$ is a $\sigma$-compact subset of $G_{\tau} / H$. Then we have $\Phi \mu \in M\left(G_{\tau} / H\right)$ by Proposition 5 . Let $\nu \in M\left(G_{\tau} / H\right)$, then there exists a Borel set $C_{1}$ of $G_{\tau_{0}} / H$ such that $\psi^{-1}\left(C_{1}\right)$ is $\sigma$-compact and $\nu$ is concentrated on $C_{1}$. There exists $\lambda \in M\left(G_{\tau_{0}}\right)$ such that $\Phi \lambda=\nu$ by Proposition 3. We put $\lambda_{0}(E)=\lambda\left(E \cap \varphi_{1}^{-1}\left(C_{1}\right)\right)$ for every Borel set $E$ of $G_{\tau_{0}}$. Then we have $\Phi \lambda_{0}=\nu$. Since $\eta^{-1}\left(\varphi_{1}^{-1}\left(C_{1}\right)\right)=\varphi_{2}^{-1}\left(\psi^{-1}\left(C_{1}\right)\right)$, $\eta^{-1}\left(\varphi_{1}^{-1}\left(C_{1}\right)\right)$ is a $\sigma$-compact subset of $G_{\tau}$ by Proposition 4. By Proposition 5 , we have $\lambda_{0} \in M\left(G_{\tau}\right)$. Then $\Phi\left(M\left(G_{\tau}\right)\right)=M\left(G_{\tau} / H\right)$. Next, we shall show (8). For every Borel set $C_{2}$ of $G_{\tau_{0}} / H$ such that $\psi^{-1}\left(C_{2}\right)$ is a $\sigma$-compact subset of $G_{\tau} / H, \eta^{-1}\left(\varphi_{1}^{-1}\left(C_{2}\right)\right)$ is $\sigma$-compact. Then $\Phi \mu\left(C_{2}\right)=\mu\left(\varphi^{-1}\left(C_{2}\right)\right)=0$ for every $\mu \in M\left(G_{\tau}\right)^{\perp}$. By Corollary 6, we have $\Phi \mu \in M\left(G_{\tau} / H\right)^{\perp}$. Conversely, for $\nu \in M\left(G_{\tau} / H\right)^{\perp}$, there exists $\mu \in M\left(G_{\tau_{0}}\right)$ such that $\Phi \mu=\nu$. By Proposition 6 , we have $\mu=\mu_{1}+\mu_{2}$ where $\mu_{1} \in M\left(G_{\tau}\right)$ and $\mu_{2} \in M\left(G_{\tau}\right)^{\perp}$. Since $\Phi \mu_{1} \in$ $M\left(G_{\tau} / H\right)$ and $\Phi \mu_{2} \in M\left(G_{\tau} / H\right)^{\perp}$, we have $\Phi \mu_{2}=\nu$. Then $\Phi\left(M\left(G_{\tau}\right)^{\perp}\right)=M\left(G_{\tau} / H\right)^{\perp}$. 
The following lemma is essential to show our main theorem.

LEMмA 9. Let $K$ be a $\sigma$-compact open subgroup of $G_{\tau}$. Then there exists a compact subgroup $H$ of $G_{\tau}$ such that $G_{\tau_{0}} / H$ contains a perfect compact $(\varphi(K), 1)$-independent subset, where $\varphi$ is the canonical map from $G_{\tau_{0}}$ onto $G_{\tau_{0}} / H$.

Proof. Let $K=\bigcup_{m=1}^{\infty} K_{m}$, such that $K_{1} \subset K_{2} \subset, \cdots, K_{m} \subset \cdots(m=$ $1,2, \cdots)$ are compact subsets of $G_{\tau}$. There exists a countable family $\left\{U_{n}\right\}(n=1,2, \cdots)$, where $U_{n}$ is a compact neighborhood of $0 \in G_{\tau}$ such that

(9) $U_{n}=-U_{n}(n=1,2, \cdots)$,

(10) $U_{n} \supset U_{n+1}+U_{n+1}(n=1,2, \cdots)$.

Let $K_{0}=\bigcap_{n=1}^{\infty} U_{n}$, then $K_{0}$ is a compact subgroup of $G_{\tau}$. By Proposition 3 of [1], there exists a countable family $\left\{W_{m, n}\right\}(m, n=1,2, \cdots)$, where $W_{m, n}$ is a compact neighborhood of $0 \in G_{\tau_{0}}$ such that

(11) $W_{m, n}=-W_{m, n}$,

(12) $W_{m, n} \supset W_{m, n+1}+W_{m, n+1}$, and

(13) $W_{m, n} \cap K_{m} \subset U_{m}$.

Let $V_{n}=\bigcap_{j=1}^{n} \bigcap_{k=1}^{n} W_{j, k}$, then $\left\{V_{n}\right\}(n=1,2, \cdots)$ has the following properties:

(14) $V_{n}=-V_{n}$,

(15) $V_{n} \supset V_{n+1}+V_{n+1}$, and

(16) $V_{n} \cap K_{n} \subset U_{n}(n=1,2, \cdots)$.

Let $H_{0}=\bigcap_{n=1}^{\infty} V_{n}$, then $H_{0}$ is a compact subgroup of $G_{\tau_{0}}$. For $x \in H_{0} \cap K$, there exists a positive integer $n_{0}$ such that $x \in K_{n} \cap V_{n}$ for every $n \geqq n_{0}$. Since $U_{1} \supset U_{2} \supset \cdots$, we have $x \in K_{0}$. Thus we get that $H_{0} \cap K \subset K_{0}$, and $H_{0} \cap K$ is a compact subgroup of $G_{\tau}$. Let $\varphi_{0}$ be the canonical map from $G_{\tau_{0}}$ onto $G_{\tau_{0}} / H_{0} \cap K$, then

(17) $\varphi_{0}\left(H_{0}\right) \cap \varphi_{0}(K)=\{0\}$.

We consider the following two cases.

Case I. Suppose $\varphi_{0}\left(H_{0}\right)$ is an infinite compact subgroup of $G_{\tau_{0}} / H_{0} \cap K$. Then there exists a perfect independent set of $\varphi_{0}\left(H_{0}\right)$. By (17), it is a $\left(\varphi_{0}(K), 1\right)$-independent set. Thus $H=H_{0} \cap K$ and $\varphi=\varphi_{0}$ satisfy this lemma.

Case II. Suppose $\varphi_{0}\left(H_{0}\right)$ is a finite compact subgroup of $G_{\tau_{0}} / H_{0} \cap K$. Let $\varphi_{1}$ be the canonical map from $G_{\tau_{0}}$ to $G_{\tau_{0}} / H_{0}$. Since $\varphi_{0}\left(H_{0}\right)$ is finite, $H_{0}$ is a compact subgroup of $G_{\tau}$. Now, we show $\varphi_{1}(K)$ is a set of the first category in $G_{\tau_{0}} / H_{0}$. Otherwise there exists a positive integer $n$ such that $\varphi_{1}\left(K_{n}\right)$ contains an interior point. Since $\varphi_{1}^{-1}\left(\varphi_{1}\left(K_{n}\right)\right)=K_{n}+H_{0}, K_{n}+H_{0}$ contains an interior point in $G_{\tau_{0}}$. Then we have $m_{\tau_{0}}\left(K_{n}+H_{0}\right)>0$, where $m_{\tau_{0}}$ is a Haar measure on $G_{\tau_{0}}$. Since $K_{n}+H_{0}$ is a compact subset of $G_{\tau}$, 
by Proposition 5 we have $m_{\tau_{0}}\left(K_{n}+H_{0}\right)=0$, a contradiction. Since $G_{\tau_{0}} / H_{0}$ is metrizable $([2])$, there is a $\left(\varphi_{1}(K), 1\right)$-independent compact perfect subset of $G_{\tau_{0}} / H_{0}$ ([10]). We put $H=H_{0}$ and $\varphi=\varphi_{1}$, then the proof is complete.

q.e.d.

THEOREM 10. Let $\tau$ be a locally compact abelian group topology on $G$ strictly stronger than $\tau_{0}$, then we have $M(\Delta) \subset M\left(G_{\tau}\right)^{\perp}$.

Proof. Since $M(\Delta)$ and $M\left(G_{\tau}\right)$ are $L$-ideals, it is sufficient to show that for any non-negative $\mu \in M\left(G_{\tau}\right)$, we have $\mu \notin M(\Delta)$. Let $K$ be a $\sigma$ compact open subgroup of $G_{\tau}$. We take $H$ and $\varphi$ satisfying Lemma 9 . Let $\Phi$ be the homomorphism from $M\left(G_{\tau_{0}}\right)$ to $M\left(G_{\tau_{0}} / H\right)$ induced by $\varphi$. Let $\mathfrak{F}$ be the Raikov system generated by $\varphi(K)$, then $M(\mathfrak{F})=M\left(G_{\tau} / H\right)$. By Proposition 8 and Theorem 1, for any nonzero $\mu \in M\left(G_{\tau}\right)$, there exists a non-symmetric complex homomorphism $f$ on $M\left(G_{\tau_{0}} / H\right)$ such that $f \circ \Phi(\mu)=f(\Phi \mu) \neq 0$. From (c) of Proposition $3, f \circ \Phi$ is a nonsymmetric homomorphism $f$ on $M\left(G_{\tau_{0}}\right)$. Thus we have $\mu \notin M(\Delta)$.

q.e.d.

3. Gelfand transforms of $M(\Delta)$. Let $G$ be a nondiscrete locally abelian group, and $S$ be Taylor's structure semigroup of $M(G)$. The maximal ideal space of $M(G)$ is identified with $\hat{S}$, with the weak*-topology of $M(G)$, the set of all nonzero continuous semicharacters on $S$. We may consider $\hat{S}$, a compact separately continuous abelian semigroup. Let $H=\left\{f \in \widehat{S}:|f|^{2}=|f|\right\}$, then $\widehat{S} \backslash H \neq \varnothing$ (c.f. [7]).

B. E. Johnson [4] showed that $(\hat{S} \backslash H) \cap \Delta \neq \varnothing$. In this section, we give a topological characterization of $(\hat{S} \backslash H) \cap \Delta$. For $f \in \hat{S} \backslash H$, we put $J(f)=\{x \in S: f(x)=0\}$ and $\mathfrak{M}(J(f))=\{\mu \in M(G): \operatorname{supp} \theta \mu \subset J(f)\}$. Let $C$ be the complex field and $C^{+}=\{z \in C: \operatorname{Re} z>0\}$.

THEOREM 11. $\hat{S} \backslash H$ is contained in the weak*-closure of $\hat{S} \backslash \Delta$ in $\hat{S}$, that is $\overline{\hat{S} \backslash \Delta} \supset \hat{S} \backslash H$.

Proof. Let $f \in \hat{S} \backslash H$ and $f \in \Delta$. Then there exists $h_{f} \in H$ such that $f=h_{f}|f|$ by the polar decomposition theorem ([7]). We put $f_{z}=h_{f}|f|^{z}$ for $z \in C^{+}$, then $f_{z} \in \hat{S}$. Let $V$ be any neighborhood of $f$. We may assume that

$$
V=\left\{g \in \hat{S}:\left|\hat{\mu}_{i}(f)-\hat{\mu}_{i}(g)\right|<\varepsilon, \mu_{i} \in M(G), i=1,2, \cdots, n\right\} .
$$

Since $f_{z} \rightarrow f(z \rightarrow 1)$ is uniformly convergent, there exists $\delta>0$ such that $f_{z} \in V$ for $z \in\left\{x \in C^{+}:|1-x|<\delta\right\}$. Since $f \in \hat{S} \backslash H$, there exists $x_{0} \in S$ such that $0<\left|f\left(x_{0}\right)\right|<1$. We take a neighborhood $U\left(x_{0}\right)$ of $x_{0}$ such that $0<$ $|f(x)|<1$ on $U\left(x_{0}\right)$. The image of $M(G)$ is weak ${ }^{*}$-dense in $M(S)$, then there exists $\mu \in M(G)$ such that the support of $\theta \mu$ is contained in $U\left(x_{0}\right)$ 
and $\hat{\mu}(f) \neq 0$. We put $F(z)=\hat{\mu}\left(f_{z}\right)$ for $z \in C^{+}$. Then $F(z)$ is a nonconstant analytic function on $C^{+}$. Suppose that $\left\{f_{z}:|1-z|<\delta\right\} \subset \Delta$. Then we have $\overline{F(z)}=\overline{\hat{\mu}\left(f_{z}\right)}=\hat{\mu}^{*}\left(f_{z}\right)$ for $z \in\left\{x \in C^{+}:|1-x|<\delta\right\}$, and $F(z)$ is an analytic function on $\left\{x \in C^{+}:|1-x|<\delta\right\}$. Thus $\overline{F(z)}$ is a constant on $\left\{x \in C^{+}:|1-x|<\delta\right\}$. By identity theorem, $F(z)$ is a constant function on $C^{+}$. This is a contradiction. Then there exists $g \in\left\{f_{z}:|1-z|<\delta\right\}$ such that $g \notin \Delta$.

q.e.d.

Corollary 12. If $\mu \in M(\Delta)$, then $\hat{\mu}(f)=0$ for all $f \in \hat{S} \backslash H$.

Let $\hat{G}$ be the dual group of $G$. T. Shimizu ([6]) showed that

$$
(\hat{S} \backslash \Delta) \cdot \hat{G} \subset(\hat{S} \backslash \Delta) \text {. }
$$

Since $\hat{S}$ is a separetely continuous topological semigroup, we have

$$
\overline{(\hat{S} \backslash \Delta)} \cdot \hat{G} \subset \overline{(\hat{S} \backslash \Delta)} \text {. }
$$

CoRollary 13. If $f \in \hat{S} \backslash H$, then $M(\Delta) \subset \mathfrak{M}(J(f))$.

Proof. Since $f \in \overline{\hat{S} \backslash \Delta}$, we have $f \cdot \hat{G} \subset \overline{\hat{S} \backslash \Delta}$. Let $\mu \in M(\Delta)$, then we have $\hat{\mu}(g)=0$ for $g \in f \cdot \hat{G}$. This shows that $\mu \in \mathfrak{M}(J(f))$ by Shimizu ([6]). Thus we have $M(\Delta) \subset \mathfrak{M}(J(f))$.

q.e.d.

\section{REFERENCES}

[1] C. Dunkl and D. Ramirez, Bounded projections on Fourier-Stieltjes transforms, to appear.

[2] E. Hewitt AND K. A. Ross, Abstract harmonic analysis I, Springer-Verlag, 1963.

[3] J. INOUE, Some closed subalgebras of measure algebras and a generalization of P. J. Cohen's theorem, J. Math. Soc. Japan, 23 (1971), 278-294.

[4] B. E. Johnson, Symmetric maximal ideals in $M(G)$, Proc. Amer. Math. Soc., 18 (1967), 1040-1045.

[5] W. RUDIN, Fourier analysis on groups, Interscience Publishers, New York, 1962.

[6] T. Shimizu, $L$-ideals of measure algebras, Proc. Japan Acad., 48 (1972), 172-176.

[7] J. L. TAYLOR, The structure of convolution measure algebras, Trans. Amer. Math. Soc., 119 (1965), 150-166.

[8] $L$-subalgebras of $M(G)$, Trans. Amer. Math. Soc., 135 (1969), 105-113.

[9] J. H. Williamson, Banach algebra elements with idempotent powers and theorem of Wiener-Pitt Type, Function algebra, Chicago (1966), 186-198.

[10] - Raikov systems and the pathology of $M(G)$, Studia Math., 31 (1968), 399-409.

[11] Raikov systems, Symposia on theoretical physics and mathematics, 8 (1968), 173-183.

Department of Mathematics

TOKYO UNIVERSITY OF EDUCATION

TOKYO, JAPAN

AND

INSTITUTE OF APPLIED ELECTRICITY

HOKKAIDO UNIVERSITY

SAPPRO, JAPAN 